\title{
Hospital acquired native valve endocarditis: analysis of 22 cases presenting over 11 years
}

\author{
C C Lamas, S J Eykyn
}

\begin{abstract}
Objective-To analyse hospital acquired infective endocarditis cases with respect to age, sex, clinical, laboratory, and echocardiographic features, predisposition, complications, surgery, mortality, and diagnostic criteria.

Design-Prospective cohort study.

Setting-Teaching hospital.

Patients-A series of 200 patients with infective endocarditis presenting over 11 years, 168 with native valve infective endocarditis, of whom 22 acquired this infection in hospital.

Results-22 (14\%) of the 168 cases of native valve infection were hospital acquired. The most common pathogens were staphylococci $(77 \%)$. Two thirds of patients had no cardiac predisposition; one third had end stage renal disease. The most common source of infection was vascular access sites (73\%). Eleven patients died. In 11 cases, infective endocarditis was proven pathologically (six at necropsy, five during surgery) and analysis of these showed that $45 \%$ were classed as probable by the Beth Israel criteria, $73 \%$ as definite by the Duke criteria, and $\mathbf{9 1 \%}$ as definite by our suggested modifications of the Duke criteria. Figures for the 11 cases not proven pathologically were $27 \%, 73 \%$, and $91 \%$, respectively. Five of the 22 cases $(22 \%)$ were rejected by the Beth Israel criteria but none were rejected by the Duke criteria with or without our modifications.

Conclusions-Hospital acquired infective endocarditis is difficult to diagnose. The Duke criteria have improved diagnostic sensitivity and our modifications have improved it further. Mortality is high but has been reduced by surgery. This serious infection could, in many cases, be prevented by improved care of intravascular lines and prompt removal when obviously infected.

(Heart 1998;79:442-447)
\end{abstract}

Division of Infection, United Medical and Dental School,

St Thomas's Hospital, Lambeth Palace Road, London SE1 7EH, UK

C C Lamas

S J Eykyn

Correspondence to: Dr Eykyn.

Accepted for publication 15 January 1998
Keywords: native valve endocarditis; hospital acquired infective endocarditis; endocarditis

Hospital acquired (nosocomial) infective endocarditis has been recognised since the 1950s. Its incidence has increased in parallel with the advances in invasive procedures, especially venous catheterisation. The mortality of nosocomial endocarditis remains high and its early recognition difficult. We analysed 22 cases presenting to our hospital over 11 years to ascertain how these infections arose, and to judge them by the Beth Israel criteria,${ }^{1}$ the Duke criteria, ${ }^{2}$ and our modifications of the Duke criteria. ${ }^{3}$ This is the first time that the newly proposed criteria have been applied specifically to this group of patients.

\section{Methods}

Cases of infective endocarditis presenting to $\mathrm{St}$ Thomas's Hospital, London, between 1985 and 1996 were analysed. Since 1970 prospective records of all patients with bacteraemia have been kept in the department of microbiology, constituting a large computerised dataset. St Thomas's is an inner city hospital with 800 beds serving the local community as well as receiving referrals from district general hospitals, mainly in the southeast of England. Hospital acquired infective endocarditis has been defined as infective endocarditis occurring more than 72 hours after admission to hospital or directly related to a procedure performed in hospital within the preceding six months of admission. No cases involving prosthetic valves were included. During the study period there were 14 cases of hospital acquired, early onset prosthetic valve endocarditis but these infections were all acquired during valve surgery and their pathogenesis is well known and differs from that of hospital acquired native valve infection. There were no cases of late onset prosthetic valve endocarditis. All cases of hospital acquired infective endocarditis had positive blood cultures, or positive microscopy or culture of the excised valve material. Data were analysed with respect to age, sex, clinical, laboratory and echocardiographic features, predisposing heart disease or other conditions, complications, surgery, and mortality. Cases were analysed by criteria proposed by Durack et al (the Duke criteria) ${ }^{2}$ and by those proposed by von Reyn et al (the Beth Israel criteria). ${ }^{1}$ The Beth Israel criteria rely on pathological evidence from surgery or necropsy for a definite diagnosis of infective endocarditis and does not include echocardiographic findings. These criteria have been superseded by the Duke criteria that enable a definite diagnosis of infective endocarditis to be made either on pathological or clinical criteria. The two main clinical criteria are microbiological (positive blood cultures, particularly, though not exclusively, with an organism typical of infective endocarditis such as a viridans streptococcus or community acquired Staphylococcus aureus) and echocardiographic. Minor clinical criteria include predisposing cardiac condition, intravenous drug abuse, fever, and vascular and immunological phenomena. We recently proposed additional minor criteria to the Duke criteria and these have increased diagnostic sensitivity. ${ }^{3}$ 


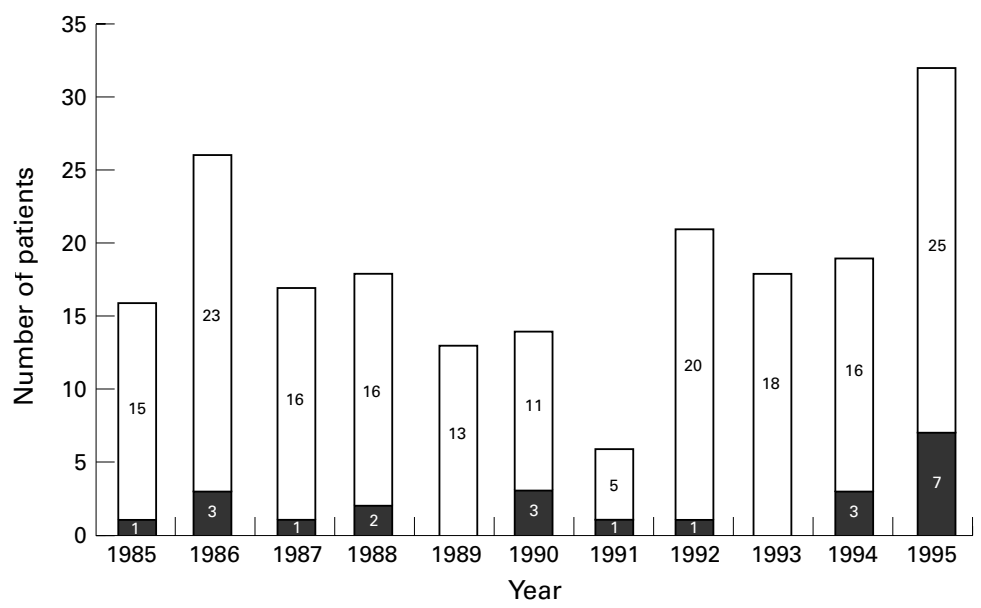

Figure 1 Annual incidence of infective endocarditis in St Thomas's Hospital 1985-95. Filled areas are hospital acquired cases.

PROPOSED ADDITIONAL MINOR CRITERIA TO THE DUKE CLASSIFICATION

- Newly diagnosed splenomegaly

- Newly diagnosed clubbing

- Splinter haemorrhages

- Petechiae

- High erythrocyte sedimentation rate (ESR), defined as more than 1.5 times the upper limit of normal (>30 $\mathrm{mm}$ in the first hour for patients younger than 60 years and $>50 \mathrm{~mm}$ in the first hour for those older than $60^{11}$ )

- High $\mathrm{C}$ reactive protein (CRP), defined as $>100 \mathrm{mg} / \mathrm{l}^{11}$

- Microscopic haematuria (disregarded when there was a positive urine culture, in menstruating women, patients with end stage renal disease, urinary catheters or urological disease)

- Central non-feeding venous lines

- Peripheral venous lines.
Pathological evidence for infective endocarditis was based on appearances of valves at surgery or necropsy, ${ }^{4}$ and microscopy and culture of valve material or embolic lesions, or histology. Results are expressed as mean (SD) or as percentages.

\section{Results}

There were 200 episodes of infective endocarditis, of which 162 were native valve infections; of these $22(14 \%)$ were hospital acquired (fig 1 ). In 1985, one of 16 cases of infective endocarditis was hospital acquired, compared with seven of 32 cases in 1995. The increased incidence of infective endocarditis probably reflects the increasing number of patients referred to St Thomas's for surgery.

The incidence of hospital acquired bacteraemia also increased over the study period: in 1985 there were 155 cases of which $23 \%$ were associated with infected intravenous access sites, and in 1995 there were 310 cases of which $54 \%$ were associated with infected intravenous access sites. Therefore, there has been a doubling of both the incidence of hospital acquired bacteraemia and the proportion caused by infected intravenous access sites.

Table 1 shows details of the 22 cases of hospital acquired infective endocarditis. There were 16 male (mean (SD) age 51.4 (25.5) years) and six female (aged 56.3 (28.5) years) patients, ranging in age from three weeks to 80 years. Twelve patients were 60 years or older and three were six months or younger. In two patients infective endocarditis was diagnosed only at necropsy. Although St Thomas's Hospital is a tertiary referral centre, only six of the 22 cases were patients referred (already infected) from another hospital.

Table 1 Details of 22 patients with hospital acquired infective endocarditis

\begin{tabular}{|c|c|c|c|c|c|c|c|c|c|}
\hline Case & Sex/age & Organism & Valve & $\begin{array}{l}\text { Cardiac } \\
\text { predisposition }\end{array}$ & Source of organism & Underlying illness & Echocardiography & Surgery & Outcome \\
\hline 1 & $\mathrm{~F} / 56$ & $S$ aureus & M & None & Arteriovenous fistula & Renal failure & TOE,minor & MVR & Survived \\
\hline 2 & $\mathrm{M} / 68$ & MRSA & M & None & Sternal wound ( 4 weeks) & $\begin{array}{l}\text { Coronary heart } \\
\text { disease }\end{array}$ & Not done & No & Died \\
\hline 3 & $\mathrm{M} / 77$ & MRSA & $\mathrm{T}$ & None & IV cannulae (1 week) & None & TTE, veg & TVR & Survived \\
\hline 4 & $\mathrm{M} / 38$ & MRSA & $\mathrm{M}$ & None & TPN line ( 2 weeks) & Appendectomy & TTE, veg & MVR & Survived \\
\hline 5 & $\mathrm{M} / 72$ & MRSA & M & None & IV cannulae $\star$ & Erythroderma & Not done & No & Died \\
\hline 6 & $\mathrm{M} / 37$ & $S$ aureus & $\mathrm{T}$ & VSD, previous IE & Arteriovenous fistula & Renal failure & TTE, NAD & No & Survived \\
\hline 7 & $\mathrm{M} / 58$ & $S$ aureus & A & Bicuspid AV & Angioplasty (3 weeks) & $\begin{array}{l}\text { Coronary heart } \\
\text { disease }\end{array}$ & TTE, veg/AR & AVR & Survived \\
\hline 8 & $\mathrm{M} / 63$ & $S$ aureus & M & None & Laminectomy wound ( 2 weeks) & None & Not done & No & Died \\
\hline 9 & $\mathrm{M} / 31$ & $S$ aureus & $\mathrm{T}$ & None & Hickman line ( 6 months) & AIDS & TTE, NAD & No & Survived \\
\hline 10 & $\mathrm{M} / 35$ & $S$ aureus & $\mathrm{T}$ & None & Central line ( 3 days) & Renal failure & Not done & No & Survived \\
\hline 11 & $\mathrm{M} / 78$ & $S$ aureus & $\mathrm{M}$ & None & Wound ( 6 weeks) & Melanoma & TTE, MR & No & Died \\
\hline 12 & $\mathrm{M} / 70$ & $S$ aureus & ? & None & IV cannulae ${ }^{\star}$ ( 2 weeks $)$ & Renal failure & TTE, NAD & No & Died \\
\hline 13 & $\mathrm{~F} / 64$ & $S$ aureus & A & $\mathrm{AR}$ & Central line ( 3 days) & Renal failure & TTE, veg & No & Died \\
\hline 14 & $\mathrm{M} / 60$ & Ent faecalis & A & None & Cystectomy (3 weeks) & Bladder cancer & TOE, veg & $\begin{array}{l}\text { AVR } \\
\text { MV repair }\end{array}$ & Survived \\
\hline 15 & $\mathrm{~F} / 74$ & Ent faecalis & M & MS & Cystoscopy (6 weeks) & Renal calculi & TOE, veg & No & Survived \\
\hline 16 & $\mathrm{~F} / 3$ weeks & $C$ albicans & $\mathrm{T}$ & PDA & TPN line ( 2 weeks) & Prematurity & TTE, veg & No & Died \\
\hline 17 & $\mathrm{M} / 3$ weeks & $C$ albicans & $\mathrm{T}$ & None & TPN line ( 2 weeks $)$ & Prematurity & TTE, veg & No & Died \\
\hline 18 & $\mathrm{M} / 6$ months & Calbicans & $\mathrm{T}$ & None & Hickman TPN line ( 2 months) & Jejunal atresia & TTE, TR & No & Died \\
\hline 19 & $\mathrm{~F} / 68$ & S epidermidis & A & MR & Permacath & Renal failure & TOE, abscess & AVR & Survived \\
\hline 20 & $\mathrm{M} / 54$ & $S$ epidermidis & M & None & Central line & Renal failure & TTE, minor & No & Died \\
\hline 21 & $\mathrm{M} / 80$ & $S$ epidermidis & A & Bicuspid AV & IV cannulae ( 3 weeks) & Bladder cancer & TTE, veg & No & Died \\
\hline 22 & $\mathrm{~F} / 76$ & $S$ epidermidis & A & None & Central line ( 3 months) & Polya gastrectomy & TTE, veg & No & Survived \\
\hline
\end{tabular}

^Peripheral.

MRSA, methicillin resistant $S$ aureus; $\mathrm{M}$, mitral; A, aortic; T, tricuspid; ?, not known; AR, MR, TR, aortic, mitral, tricuspid regurgitation; PDA, persistent ductus arteriosus; VSD, ventricular septal defect; IE, infective endocarditis; IV, intravenous; TPN, total parenteral nutrition; AV, arteriovenous; TTE, TOE, transthoracic, transoesophageal echocardiography; veg, vegetation; NAD, no abnormality detected; MV, mitral valve; AVR, TVR, MVR, aortic, tricuspid, mitral valve replacement. 
Table 2 Hospital acquired bacteraemia $(n=2514)$ and endocarditis $(n=22)$

\begin{tabular}{lll}
\hline Bacteria causing endocarditis & $\begin{array}{l}\text { Episodes of } \\
\text { bacteraemia }\end{array}$ & $\begin{array}{l}\text { Episodes of } \\
\text { endocarditis }\end{array}$ \\
\hline S aureus (not MRSA) & 402 & $9(2 \%)$ \\
MRSA & 47 & $4(8.5 \%)$ \\
Coagulase negative staphylococci & 376 & $4(1 \%)$ \\
Enterococci & 195 & $2(1 \%)$ \\
Candida spp & 60 & $3(5 \%)$ \\
\hline
\end{tabular}

MRSA, methicillin resistant $S$ aureus.

CAUSATIVE ORGANISMS

Seventeen of 22 cases were caused by staphylococci ( $S$ aureus 13 cases, $S$ epidermidis four cases). Four of the 13 isolates of $S$ aureus were methicillin resistant (MRSA). The three Candida albicans infections occurred in children younger than six months. Table 2 shows the absolute numbers of hospital acquired bacteraemia and of hospital acquired endocarditis over the 11 year study period, and the causative organisms.

\section{VALVES AFFECTED}

The mitral valve was affected in eight patients, the aortic in six, and the tricuspid in seven. In one patient the affected valve could not be determined.

PREDISPOSING HEART DISEASE OR OTHER

CONDITIONS

Fifteen of 22 patients had no intrinsic predisposing heart disease; table 1 shows the conditions present in the other seven patients. Seventeen of the 22 cases had a vascular non-cardiac predisposition: 10 central intravenous catheters (four feeding lines), six peripheral intravenous cannulae, and four arteriovenous fistulae.

\section{MODE OF ACQUISITION OF INFECTIVE}

ENDOCARDITIS

Infection of vascular access sites accounted for most cases. Other sources of infection were the urinary tract and wounds (table 1). The same strain of $S$ aureus was isolated from sites other than blood in six of 13 cases of infective endocarditis (line tips four cases, wounds two cases), and in two of the three cases of $C$ albicans infection (both line tips).

\section{UNDERLYING ILLNESS}

Six patients had end stage renal disease and were on haemodialysis. Two were premature babies (25 and 31 weeks' gestation, respectively). The six month old baby boy with jejunal atresia had been on total parenteral nutrition since birth and had had a Hickman line inserted two months before his infection.

\section{CLINICAL FEATURES}

Table 3 shows the clinical feature of the 22 patients; splenomegaly, Osler's nodes, Janeway lesions, and Roth's spots were not seen.

ECHOCARDIOGRAPHIC FEATURES

Transthoracic echocardiography was performed in 18 patients: vegetations were detected in eight, new valvar regurgitation in two, and valve thickening in one. Transthoracic
Table 3 Clinical features of 22 patients with hospital acquired infective endocarditis

\begin{tabular}{ll}
\hline Clinical feature & Number (\%) \\
\hline Fever & \\
$>38.9^{\circ} \mathrm{C}$ & $10(45)$ \\
$38-38.9^{\circ} \mathrm{C}$ & $10(45)$ \\
$<38^{\circ} \mathrm{C}$ & $1(5)$ \\
Not known & $1(5)$ \\
Malaise & $8(36)$ \\
Anorexia & $1(5)$ \\
Chills & $3(14)$ \\
Weight loss & $2(9)$ \\
Night sweats & $1(5)$ \\
Back pain & $2(9)$ \\
Non-specific vascular phenomena & $4(18)$ \\
Clubbing & $1(5)$ \\
New regurgitant murmur & $7(32)$ \\
Dyspnoea & $4(18)$ \\
Pleuritic chest pain & $2(9)$ \\
Change in mental state & $5(23)$ \\
\hline
\end{tabular}

^Includes rashes, petechiae, and splinter haemorrhages.

echocardiography was not helpful in the other seven patients, four of whom had transoesophageal echocardiography: two had vegetations, one an abscess, and one a perforated valve leaflet. Thus, major echocardiographic criteria ${ }^{2}$ were detected in $56 \%$ of transthoracic echocardiograms and minor echocardiographic criteria ${ }^{2}$ in $6 \%$.

\section{LABORATORY FEATURES}

Haemoglobin concentrations and leucocyte counts were not analysed because of confounding factors including recent surgery and underlying illnesses. ESR was determined for eight patients and was high in seven (41$150 \mathrm{~mm}$ in the first hour). CRP concentrations measured in five patients were $65,100,111$, 125 , and $297 \mathrm{mg} / \mathrm{l}$. Urine microscopy was performed in 16 patients but 11 were excluded from analysis (end stage renal disease, two patients; positive urine cultures, two; renal stones, one; and carcinoma of the bladder, two). Microscopic haematuria was detected in four of the other five patients.

\section{COMPLICATIONS AND MORTALITY}

Table 4 shows the complications and table 1 the mortality of the 22 patients. The six patients who had valve replacement surgery all survived, but 11 of the 16 who did not have surgery died. Three children with $C$ albicans infection and six elderly patients with $S$ aureus died. Three of the five patients who survived without surgery were in their 30s and had $S$ aureus infective endocarditis of the tricuspid valve.

\section{DIAGNOSTIC CRITERIA}

Table 5 shows the clinical assessment of the cases. The 11 pathologically proven cases were assessed using the clinical criteria to test the

Table 4 Complications in 22 cases of hospital acquired infective endocarditis

\begin{tabular}{ll}
\hline Complication & Number (\%) \\
\hline Persistent fever & $6(27)$ \\
Brain abscess/emboli & $5(23)$ \\
Heart failure & $4(18)$ \\
Septic pulmonary emboli & $4(18)$ \\
Valvar/myocardial abscess & $2(9)$ \\
Splenic abscess & $3(14)$ \\
Renal failure (dialysis necessary) & $1(5)$ \\
None of the above & $3(14)$ \\
\hline
\end{tabular}


Table 5 Clinical assessment of 22 cases of hospital acquired infective endocarditis

\begin{tabular}{llll}
\hline Criteria/clinical assessment & $\begin{array}{l}\text { Pathologically proven } \\
(n=11)\end{array}$ & $\begin{array}{l}\text { Not pathologically } \\
\text { proven }(n=11)\end{array}$ & Total $(n=22)$ \\
\hline Beth Israel & $5(45 \%)$ & $3(27 \%)$ & $8(36 \%)$ \\
$\quad$ Probable & 2 & 7 & $9(41 \%)$ \\
Possible & 4 & 1 & $5(23 \%)$ \\
$\quad$ Rejected & $8(73 \%)$ & $8(73 \%)$ & $16(73 \%)$ \\
Duke & 3 & 3 & $6(27 \%)$ \\
$\quad$ Definite & 0 & 0 & 0 \\
Possible & $10(91 \%)$ & $10(91 \%)$ & $20(91 \%)$ \\
$\quad$ Rejected & 1 & 1 & $2(9 \%)$ \\
Duke with modifications & 0 & 0 \\
$\quad$ Definite & 0 & 0 & \\
Possible & & & \\
Rejected & & & \\
\hline
\end{tabular}

different diagnostic criteria. The diagnosis of definite infective endocarditis by the Duke criteria was made on the basis of two major criteria in three of eight cases in the pathologically proven group, and in six of eight cases in the non-pathologically proven group. No definite diagnosis was made on the basis of five minor criteria. Our suggested modifications ${ }^{3}$ upgraded four possible cases of infective endocarditis as defined by the Duke criteria to definite: by adding one minor criterion to one major and two minor in three cases; and by adding two minor criteria to one major and one minor in another case. No cases were rejected by the Duke criteria or by our modifications, but five cases were rejected by the Beth Israel criteria.

\section{Discussion}

The reported incidence of hospital acquired infective endocarditis ranges from $13-17 \%$ of episodes of infective endocarditis in series that included early prosthetic infective endocarditis, ${ }^{5-7}$ and from $9.3-20 \%$ in those that included only native valves. ${ }^{89}$ Our incidence of $14 \%$ included only native valves. Hospital acquired infective endocarditis has increased sevenfold over the past 11 years in our institution.

In common with other series ${ }^{5-79}$ the most common pathogen was $S$ aureus, which accounted for over half the cases. One third of the $S$ aureus isolates were MRSA, a similar incidence to that reported by Chen and colleagues $^{7}$; however, Fernandez-Guerrero and colleagues $^{9}$ had no MRSA in their series. In our institution, over the 11 year period, $3 \%$ of patients with $S$ aureus hospital acquired bacteraemia and $8.5 \%$ with MRSA bacteraemia had hospital acquired infective endocarditis $(p=0.037$, Fisher's test), which is an interesting observation as MRSA bacteraemia may be an indirect marker of prolonged hospitalisation and antibiotic use.

Coagulase negative staphylococci are well recognised pathogens in venous access infections, and the reported incidence of these organisms in hospital acquired infective endocarditis ranges from 3-7\%, ${ }^{79}$ although it is inexplicably high $(64 \%)$ in the series reported by Terpennning et al. ${ }^{6}$ Only $1 \%$ of our cases of hospital acquired bacteraemia with coagulase negative staphylococci had endocarditis.
Enterococci caused $9 \%$ of our cases; in other series they were responsible for $10-30 \%$ of cases..$^{5-9}$ None of our isolates was resistant to vancomycin or showed high level gentamicin resistance. In the study period, 195 episodes of hospital acquired bacteraemia were caused by enterococci; therefore, endocarditis occurred in $1 \%$ of cases, a similar finding to that reported by Maki and Agger. ${ }^{10}$

$C$ albicans was the only organism isolated in the paediatric cases in this series, and was associated with central feeding lines in all three cases. This has been noted in a recent paediatric series ${ }^{11}$ in which six of 13 cases of hospital acquired infective endocarditis were caused by $C$ albicans.

We have expanded the non-cardiac predispositions for infective endocarditis as defined by Durack ${ }^{12}$ (central feeding lines and arteriovenous fistulae) to include central non-feeding lines and peripheral cannulae. In common with other series, ${ }^{679}$ two thirds (15 of 22) of our patients had no intrinsic cardiac predisposition for infective endocarditis, and of these 15 patients, 10 acquired infective endocarditis by infected venous access (central feeding line, three; central non-feeding line, four; peripheral line, two; arteriovenous fistula, two). Central intravenous catheters are known to damage normal endothelium in an experimental model ${ }^{13}$ by facilitating bacterial adhesion from local seeding or, less often, from distant bacteraemia. This is also likely to occur in man and has been noted in several reports. ${ }^{14-17}$ Central intravenous catheterisation was responsible for infective endocarditis in 10 of our 22 cases, and only four of these catheters were for total parenteral nutrition. Interestingly, there were no cases related to pulmonary catheters. The reported incidence of nosocomial infective endocarditis associated with central intravenous catheters ranged from $9.1-48 \%^{5-7} 9$ but vascular access is not mentioned in the earliest paper on hospital acquired infective endocarditis. ${ }^{8}$ In that series of 17 cases of necropsy proved, hospital acquired infective endocarditis, the infection was thought to have resulted from surgical wounds (gastric and genitourinary in 11 cases) and genitourinary instrumentation (12 cases). Peripheral intravenous cannulation was widely practised at that time ${ }^{18} 19$ but it does not seem to have been recognised as a portal of entry for such infections. Peripheral intravenous catheters accounted for $6-22.7 \%$ of cases of nosocomial infective endocarditis in reported series $^{5-8}$ and for $18 \%$ of our cases. Although, in general, peripheral catheters are associated with lower infection risks than central catheters, ${ }^{20}$ the number of peripheral cannulae far exceeds that of central ones, their placement may be hasty and done by inexperienced medical personnel, and their care may be suboptimal; not surprisingly, local infection is common.

Infective endocarditis has long been recognised as a complication of maintenance haemodialysis, ${ }^{21-23}$ and has been reported in patients with arteriovenous fistulae (with or without prosthetic grafts). Arteriovenous 
fistulae seldom show overt signs of infection when bacteraemia is detected, and the repetitive needling of the skin/fistula is probably the main risk factor in these patients. They are also often subject to frequent hospital admissions where peripheral and central venous catheters are inserted, and they may be dialysed via devices such as the Permacath while their recently constructed fistula "matures". It is not surprising that seven of our 22 patients had end stage renal disease and all acquired infective endocarditis by venous access.

Nearly half of our patients presented with high grade fever but only one third had new regurgitant murmurs. This may reflect the predominance of $S$ aureus in hospital acquired infective endocarditis, and has been described previously. ${ }^{5-9} 2425$ This may also explain the neurological presentation in $25 \%$ of our cases. ${ }^{24-27}$ In patients who are already ill, these features should raise the possibility of endocarditis if no other focus of infection is recognisable and if signs of infection persist, particularly with repeatedly positive blood cultures. Non-specific vascular phenomena, although not included in the Duke criteria, ${ }^{2}$ were detected in four of our cases and, in conjunction with other features, may be a further clue to diagnosis.

Persistent fever was the most common complication in our patients with hospital acquired infective endocarditis, surpassing heart failure. Septic cerebral emboli occurred in five of our cases; Guze and Pearson ${ }^{8}$ reported a $12 \%$ incidence of "cerebrovascular events" and Chen and colleagues ${ }^{7}$ a $67 \%$ incidence of neurological complications. Septic pulmonary emboli reflected tricuspid involvement mostly related to central intravenous cannulation.

Mortality of hospital acquired infective endocarditis was $100 \%$ in the first published series $^{8}$ when cardiac surgery was not available. It still remains high although all six patients in our series who had surgery survived, whereas 11 of the 16 who did not have surgery died. The overall mortality was $50 \%$ and was directly related to infective endocarditis. Reported mortality related to the disease ranges from $40-54.5 \%$ in recent series..$^{5-7} 9$

It has been shown previously that the Beth Israel criteria have a lower sensitivity than the Duke criteria for the diagnosis of infective endocarditis ${ }^{28-30}$; we have confirmed this for prosthetic and native valve endocarditis analysed separately. ${ }^{3}$ In the clinical assessment of hospital acquired infective endocarditis, the Duke criteria diagnosed $73 \%$ of both pathologically proved and non-pathologically proved infective endocarditis as definite; our modifications increased this diagnostic yield to $91 \%$ for both groups. The Beth Israel criteria performed poorly with $45 \%$ and $21 \%$ probable diagnoses, respectively, and most importantly, they rejected five of the 22 cases while none were rejected by the Duke criteria or by our modifications.

None of the recent series of hospital acquired infective endocarditis analysed the echocardiographic aspects of the infection. Major echocardiographic criteria were detected in $56 \%$ of the transthoracic echocardiographies performed in our series, which compares with $66 \%$ for native and $44 \%$ for prosthetic valve endocarditis. $^{3}$

Hospital acquired bacteraemia has increased dramatically in the past decade with a parallel increase in hospital acquired infective endocarditis. Vascular access site infection is the main cause of both. Prevention of these infections involves educating medical and nursing staff in the placement and care of all catheters, and the close inspection of the skin entry site with the prompt removal of the device when infection is detected. We do not consider that there is any need for prophylactic antibiotics in the prevention of intravenous access site infections. In fact, the use of prophylactic antibiotics may encourage infection with more resistant organisms. Hospital acquired infective endocarditis is difficult to diagnose but the Duke criteria have improved diagnostic sensitivity compared with the Beth Israel criteria, and our modifications have improved it further. Mortality remains high but has been greatly improved by surgery.

1 von Reyn CF, Levy BS, Arbeit RD, et al. Infective endocarditis: an analysis based on strict case definitions. Ann Intern Med 1981;94:505-18.

2 Durack DT, Lukes AS, Bright DK, Duke Endocarditis Service. New diagnostic criteria for diagnosis of infective endocarditis: utilization of specific echocardiographic findings. Am F Med 1994;96:200-9

3 Lamas CC, Eykyn SJ. Suggested modifications to the Duke criteria for the clinical diagnosis of native and prosthetic valve endocarditis: analysis of 118 pathologically proven cases. Clin Infect Dis 1997;25:713-19.

4 Delahaye F, Goulet V, Lacassin F, et al. Characteristics of infective endocarditis in France in 1991. A 1-year survey. Eur Heart $\mathcal{F} 1995 ; 16: 394-401$.

5 Friedland G, von Reyn CF, Levy BS, et al. Nosocomial endocarditis. Infection Control 1984;5:284-8. 6 Terpenning MS, Buggy BP, Kauffman CA. Hospital1601-3.

7 Chen SCA, Dwyer DE, Sorrell TC. A comparison of hospital and community-acquired infective endocarditis. $A m \mathcal{F}$ Cardiol 1992;70:1449-52.

8 Guze LB, Pearce ML. Hospital-acquired bacterial endocarditis. Arch Intern Med 1963;112:56-62.

9 Fernández-Guerrero ML, Verdejo C, Azofra J, et al. Hospital-acquired infective endocarditis not associated with cardiac surgery: an emerging problem. Clin Infect Dis 1995;20:16-21

10 Maki DG, Agger WA. Enterococcal bacteremia: clinical features, the risk of endocarditis, and management. Medicine 1988;67:248-65:

11 Saiman L, Prince A, Gersony WM. Pediatric infective endocarditis in the modern era. F Pediatr 1993;122:847-53.

12 Durack DT. Prophylaxis of infective endocarditis. In: Mandell GL, Bennett JE, Dolin R, eds. Principles and practice of infectious diseases. Vol 1. New York: Churchill Livingstone, infectious diseases.

13 Contrepois A. Notes on the early history of infective endocarditis and the development of an experimental model. carditis and the development
Clin Infect Dis 1995;20:461-6.

14 Baskin TW, Rosenthal A, Pruitt BA. Acute bacterial endocarditis: a source of sepsis in the burn patient. Ann Surg 1976;184:618-21.

15 Ehrie M, Morgan AP, Moore FD, et al. Endocarditis with the indwelling balloon-tipped pulmonary artery catheter in burn patients. F Trauma 1978;18:664-6.

16 Rowley KM, Clubb KS, Walker Smith GJ, et al. Right-sided infective endocarditis as a consequence of flow-directed pulmonary catheterisation. A clinicopathological study of 55 autopsied patients. N Engl f Med 1984;311:1152-6.

17 Tsao MMP, Katz D. Central venous catheter-induced endocarditis: human correlate of the animal experimental model of endocarditis. Rev Infect Dis 1984;6:783-90.

18 Ladd M, Schreiner GE. Plastic tubing for intravenous Ladd $M$, Schreiner GE. Plastic tubing
alimentation. $尹 A M A$ 1951;145:642-3.

19 Anderson LH. Venous catheterization for fluid therapy: a Anderson LH. Venous catheterization for fluid therap
technique and results. F Lab Clin Med 1950;36:645-9.

20 Raad II, Bodey GP. Infectious complications of indwelling vascular catheters. Clin Infect Dis 1992;15:197-208. 
21 Goodman JS, Crews HD, Grin HE, et al. Bacterial endocarditis as a possible complication of chronic haemodialysis. $N$ ditis as a possible complicatio
Engl f Med 1969;280:876-7.

22 King LH, Bradley KP, Shires DL, et al. Bacterial endocarditis in chronic hemodialysis patients: a complication more common than previously suspected. Surgery 1971:69:554-6.

23 Cross AS, Steigbigel RT. Infective endocarditis and access site infections in patients on haemodialysis. Medicine 1976; 55:453-66.

24 Chambers HF, Korzeniowski OM, Sande MA, with the National Collaborative Endocarditis Study Group. Staphylococcus aureus endocarditis:clinical manifestations in addicts and nonaddicts. Medicine 1983;62:170-7.

25 Watanakunakorn C. Staphylococcus aureus endocarditis at a community teaching hospital,1980 to 1991 . An analysis of 106 cases. Arch Intern Med 1994;154:2330-5.
26 Pruitt AA. Neurologic complications of infective endocarditis:a review of an evolving disease and its management issues in the 1990s. Infect Dis Clin Pract 1996; 5:101-13.

27 Gransden WR, Eykyn SJ, Leach RM. Neurological presentations of native valve endocarditis. $Q \mathcal{F}$ Med 1989;272: $1135-42$.

28 Hoen B, Selton-Suty C, Danchin N, et al. Evaluation of the Duke criteria versus the Beth Israel criteria for the diagnosis of infective endocarditis. Clin Infect Dis 1995;21: 905-9.

29 Sandre RM, Shaffran SD. Infective endocarditis: review of 135 cases over 9 years. Clin Infect Dis 1996;22:276-86.

30 Bayer AS, Ward JI, Ginzton LE, et al. Evaluation of new clinical criteria for the diagnosis of infective endocarditis. Am F Med 1994;96:211-19. 